\title{
Mitochondrial Abnormalities at Autopsy of Fetal Noncompaction
}

\author{
Josef Finsterer $^{1 *}$ and Sinda Zarrouk Mahjoub ${ }^{2}$ \\ ${ }^{1}$ Krankenanstalt Rudolfstiftung, Austria \\ ${ }^{2}$ Department of Medicine Monastir, Tunisia \\ *Corresponding author: Josef Finsterer, Krankenanstalt Rudolfstiftung, Vienna, Austria
}

\section{Letter to the Editor}

In a recent article, Tian et al. [1] reported about the echocardiographic and pathological findings in 9 fetuses with left ventricular hyper trabeculation/non-compaction (LVHT). We have the following comments and concerns. The authors differentiate between non-compaction cardiomyopathy (NCCM) and left ventricular non-compaction (LVNC). In our view these are two different terms which describe the same entity. Which is the difference between NCCM and LVNC in the authors' view? Absence of additional cardiac abnormalities should not be a differentiating criterion.

We do not agree that ultrastructural investigations of noncompaction cardiomyopathy have not been carried out so far [1]. In a recent review we found 11 studies in which ultrastructural investigations of the compacted and non-compacted myocardium in LVHT patients have been carried out [2]. Did the authors carry out interobserver investigations? Which was the variability between the observers? How often did different investigators agree and how often disagree on the diagnosis of LVHT in the fetuses? LVHT occurs familiarly in relatives of LVHT index patients [3]. Were first degree relatives of these 9 fetuses investigated for LVHT? Was LVHT particularly present in the mothers of these 9 fetuses? We find it unethical to propose termination of a pregnancy in case the fetus is diagnosed with LVHT. LVHT is not a life-threatening disorder. The majority of LVHT patients live a satisfactory and fulfilling life over years. Though parents should be informed about the possible complications of LVHT, termination of the pregnancy should not be proposed to them.

Recently it has been reported that LVHT develops in females during pregnancy [4]. Were also the pregnant females of the 9 carriages investigated echocardiographically? The authors talk about "muscle biopsy". Do they mean biopsy of the skeletal muscle or biopsy of the myocardium? If they mean myocardium the term "muscle biopsy" is misleading. We do not agree that LVHT was first described by Engberding in 1984. In a previous study we provided evidence that LVHT was first described by Feldt et al. 1969 and possibly even earlier [5]. What happened to the two fetuses of which the pregnancy was not terminated? Are they still alive? Did they develop any complication of LVHT, such as ventricular arrhythmias, systolic dysfunction or stroke / embolism? Did postnatal echocardiography confirm the intrauterine finding of LVHT? For how long were these two patients followed-up? Did patient B in case 8 undergo autopsy? Was LVHT found on autopsy in this patient as well?

LVHT in children is most frequently due to chromosomal defects [6]. Were the 7 fetuses undergoing autopsy investigated for chromosomal aberrations? Subendocardial fibrosis is a frequent feature in the non-compacted layer of LVHT patients [7]. Was subendocardial fibrosis found in any of the 7 histologically investigated patients? Right ventricular hyper trabeculation should not be assessed since the right ventricle is physiologically hyper trabeculated. LVHT is frequently found in patients with mitochondrial disorders [8]. Were the mitochondrial changes on ultrastructural investigations attributable to primary mitochondrial dysfunction or a secondary effect? Were biochemical investigations also carried out? Were any of the fetuses tested for mtDNA mutations? Overall, this interesting case series could substantially profit from the presentation of additional data which eventually could help to clarify the still elusive pathogenesis of LVHT. 


\section{References}

1. Tian T, Liu Y, Gao L, Wang J, Sun K, et al. (2014) Isolated left ventricular noncompaction: clinical profile and prognosis in 106 adult patients. Heart Vessels 29(5): 645-652.

2. Finsterer J, Stöllberger C (2013) Ultrastructural findings in noncompaction prevail with neuromuscular disorders. Cardiology 126(4): 219-223.

3. Finsterer J, Stöllberger C, Blazek G, Sehnal E (2013) Familal left ventricular hypertrabeculation (noncompaction) is myopathic. Int J Cardiol 164(3): 312-317.

4. Gati S, Papadakis M, Papamichael ND, Zaidi A, Sheikh N, et al. (2014) Reversible de novo left ventricular trabeculations in pregnant women: implications for the diagnosis of left ventricular noncompaction in lowrisk populations. Circulation 130(6): 475-483.
5. Finsterer J, Zarrouk Mahjoub S (2013) Grant et al. 1926 did not provide the first description of left ventricular hyper trabeculation/ noncompaction. Int J Cardiol 169(4): e51-52.

6. Finsterer J (2009) Cardiogenetics, neurogenetics, and pathogenetic of left ventricular hyper trabeculation/noncompaction. Pediatr Cardiol 30(5): 659-681.

7. Ker J, Du Toit-Prinsloo L, Van Heerden WF, Saayman G (2011) Subendocardial fibrosis in left ventricular hyper trabeculation-cause or consequence? Clin Med Insights Cardiol 5:13-16.

8. Meyers DE, Basha HI, Koenig MK (2013) Mitochondrial cardiomyopathy: pathophysiology, diagnosis, and management. Tex Heart Inst J 40(4): 385-394.

\section{(C) (P) This work is licensed under Creative}

To Submit Your Article Click Here:

Submit Article

DOI: $10.32474 /$ LOJMS.2018.02.000145

Citation: Josef F, Sinda Zarrouk M. Mitochondrial Abnormalities at Autopsy of Fetal Noncompaction. LOJ Med Sci 2(4)-2018. LOJMS. 